\title{
THE NEED FOR INTEGRATING TRADITIONAL MEDICINE IN TO MODERN MEDICAL PRACTICE
}

Dear Reader,

Thomas Edison's (1847-1931) inventions helped accelerate industrialization by taking a concept and making power generation commercially feasible and widely available. Although, he was a highly successful inventor and businessman, he did have his setbacks. He acknowledged his many failures by quoting, "I have not failed, I have just found 10,000 ways that won't work. Our greatest weakness lies in giving up. The most certain way to succeed is always to try just one more time". This is also true when it comes to discovering new drugs, especially based on traditional medicine.

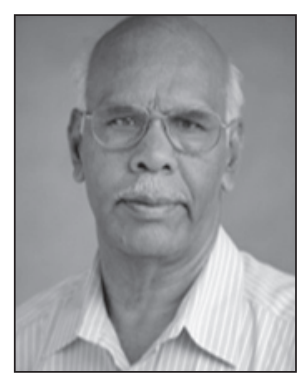

In the early years' medicinal plants were identified and used to alleviate suffering and cure diseases. These plant derived formulations became a part of folklore and what we now refer to as traditional medicine. With the advancement of technology, scientists were able to isolate, identify and characterize several active ingredients from such natural sources. Some examples include, Morphine, Codeine, Quinine, etc.

Subsequently, several anticancer agents were also reported such as Vinblastine and Vincristine from Vinca Rosea leaves. The most widely used anti-cancer drugs such as Taxol and Irinotecan are also derived from plants.

For the last two decades, natural product research, either from plants or micro-organisms, has taken a back seat with the advent of high throughput screening, combinatorial chemistry, computational chemistry, bio-informatics, etc.

In India, considerable work has been done in the search of potent natural products and substances derived from plants. Several institutes have worked in this space from 1950 - 1980 including some major institutes like NCL, Pune and CDRI, Lucknow. KEM hospital, Mumbai and CDRI have investigated and screened more than 15,000 medicinal plants. However, nothing much has come out of their work that would enable the introduction of an active molecule into the market. Nonetheless many leads have been identified but not pursued further.

Indian investigators screening Indian medicinal plants have mostly looked into major constituents which can be separated easily and purified and determined their structures. Further, chemists being specialists in certain areas such as phenolic compounds, terpenoids or alkaloids perhaps could have taken a deeper interest in a class of compounds they were more familiar with, leaving other constituents. In this process, many other active molecules could have been lost or not identified,.

Only one drug based on a natural product led to the design and synthesis of Centchroman (Ormeloxifene) discovered by the CDRI group under the direction of Dr. Nitya Anand. It is a non-steroidal oral contraceptive and is used at a dose of $30 \mathrm{mg}$ once a week. It has undergone extensive toxicological and clinical trials and proved to be safer than the alternatives. As its patents expired before it entered the Indian market, no international company had shown interest in marketing the drug. It is now produced and distributed by Hindustan Latex Ltd. Being used only once a week, it does have an advantage for women to use it as oral contraceptive.

Recently, Sun Pharmaceuticals entered into ₹240 crores global licencing deal with the Indian Institute of Chemical Technology, Hyderabad for developing new drugs from structures and NCEs that have shown exceptional therapeutic value. Among the three NCEs developed by IICT, one of them is derived from a natural product source. 
Finally, it is worthwhile to relook at the Ayurvedic drugs that have been used for centuries and many of them shown significant results for certain diseases (eg: Arthritis). It is high time for Indian industries and Government institutions to jointly work and see how best the Indian traditional system of medicine can be understood and integrated into the modern medicine to achieve better results with less expense and with minimum time. The newly formed Ministry of Ayush and UGC are providing grants to take up research projects to evaluate our traditional medicine and identify potential therapies with a clear understanding of the mechanism of action, thereby leading to the development of more potent derivatives.

The 2015 Nobel Prize in Medicine went to Ms. Youyou Tu for her work on Artemesinin that has saved several millions of lives from death due to malaria. She searched ancient Chinese texts seeking a traditional cure for fever which finally resulted in the isolation and identification of Artemesinin. Her story is truly inspiring and we should follow her model in detecting some active ingredients from natural sources. Such efforts can lead to a single compound or a mixture of products having a concoction effect as long as the science clearly explains the mode of action.

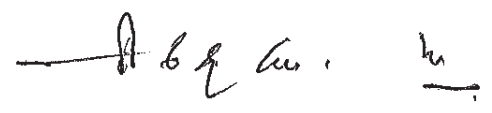

Padma Bhushan Dr. A V Rama Rao

https://doi.org/10.53879/id.56.10.p0005

\section{About the Guest Editor}

Dr A V Rama Rao is the only Fellow of the Indian National Science Academy (FNA) who has trained 112 Ph.D. students, published more than 250 papers in reputed international scientific journals, developed over 30 process technologies for making life saving drugs more affordable and finally after retiring as Director of Indian Institute of Chemical Technology, built a multi-million dollar pharmaceutical company (Avra Laboratories Pvt. Ltd.,).

$\mathrm{He}$ is an institutional builder and demonstrated his abilities by heading (1985-95) and transforming the Regional Research Laboratory into the more globally respected Indian Institute of Chemical Technology. He pioneered the concept of Institution and Industry interaction with several leading Indian pharmaceutical industries.

Dr Rama Rao has received over 45 awards including "Padma Shri" and "Padma Bhushan". He is the first Indian to receive the Technology award from the World Academy of Sciences. He rendered public service to the Government of India as the Chairman or Member of various policy making bodies especially on Drugs and Pharmaceuticals.

म

If you would like to comment on the editorial please write to us at publications@idmaindia.com 